\title{
Five-year survival rate analysis: the combination of fortnightly- administration of endostar and concurrent chemoradiotherapy versus concurrent chemoradiotherapy in the treatment of inoperable locally advanced non-small cell lung cancer
}

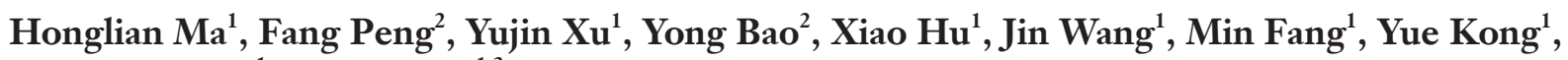 \\ Baiqiang Dong ${ }^{1}$, Ming Chen ${ }^{1,3}$
}

${ }^{1}$ The Cancer Hospital of the University of Chinese Academy of Sciences (Zhejiang Cancer Hospital), Institute of Basic Medicine and Cancer, Chinese Academy of Sciences, Hangzhou, China; ${ }^{2}$ Department of Radiation Oncology, the First Affiliated Hospital of Sun Yat-sen University, Guangzhou, China; ${ }^{3}$ Department of Radiation Oncology, the Sun Yat-sen University Cancer Center, Guangzhou, China

Contributions: (I) Conception and design: M Chen; (II) Administrative support: H Ma, F Peng; (III) Provision of study materials or patients: H Ma, F Peng, Y Xu, Y Bao, X Hu, J Wang; (IV) Collection and assembly of data: M Fang, Y Kong, B Dong; (V) Data analysis and interpretation: H Ma; (VI) Manuscript writing: All authors; (VII) Final approval of manuscript: All authors.

Correspondence to: Ming Chen. The Cancer Hospital of the University of Chinese Academy of Sciences (Zhejiang Cancer Hospital), Institute of Basic Medicine and Cancer, Chinese Academy of Sciences, Hangzhou, China. Email: Chenming@sysucc.org.cn.

Background: A prospective phase II study showed that Endostar combined with concurrent
chemoradiotherapy (CCRT) can improve overall survival (OS) in patients with inoperable locally advanced
non-small cell lung cancer (NSCLC). This study aimed to retrospectively compare the 5 -year survival rates
of patients with inoperable locally advanced NSCLC who received a combination of Endostar and CCRT to
that of patients receiving CCRT.

Methods: Treatment-naive patients with inoperable locally advanced NSCLC who had long-term followup data were included in this study. Patients in CCRT + Endostar group were treated with Endostar plus radical CCRT, and patients in CCRT group received radical CCRT. For patients with a radiation dose $\geq 60$ Gy, Kaplan-Meier method was used for survival analysis, and Cox proportional-hazards regression model was used for univariate analysis.

Results: A total of 104 participants were included in the CCRT + Endostar group with 89 participants included in the CCRT group. There were $88(84.6 \%)$ and $74(83.1 \%)$ male patients, respectively. The median follow-up times of two groups were 73.6 (95\% CI: 65.6 to 81.7 months) and 66.3 months (95\% CI: 52.7 to 79.9 months), respectively. The median overall survival (OS) was 29.7 (95\% CI: 22.8 to 36.6 months) and 21.3 months (95\% CI: 15.9 to 26.7 months), respectively.

Conclusions: This study showed that the 5-year survival of those patients who received the combination treatment of Endostar and radical CCRT was significantly superior to those who received radical CCRT.

Keywords: Non-small cell lung cancer (NSCLC); endostatin; Endostar; fortnightly administration; concurrent chemoradiotherapy (CCRT)

Submitted Apr 07, 2021. Accepted for publication Jul 13, 2021.

doi: 10.21037/apm-21-1092

View this article at: https://dx.doi.org/10.21037/apm-21-1092 


\section{Introduction}

A meta-analysis in 2010 showed that for patients with inoperable locally advanced non-small cell lung cancer (NSCLC), concurrent chemoradiotherapy (CCRT) was superior to the sequential chemoradiotherapy (SCRT) (1), making CCRT the standard treatment for inoperable locally advanced NSCLC. However, how to optimize the two treatment methods and further improve their efficacy remained to be explored (2-8). The PACIFIC study $(9,10)$ made CCRT plus durvalumab consolidation immunotherapy the new standard treatment for locally advanced NSCLC. However, the PACIFIC study conducted a high-standard screening among the participants. The enrolled patients were those who had no disease progression after CCRT, and some who were resistant to CCRT were dropped from the study. Therefore, challenges remain in how to overcome the resistance of patients with lung cancer to chemoradiotherapy and improve the efficacy of CCRT.

Tumors are obviously resistant to radiation during hypoxia, leading to failure of local tumor growth control $(11,12)$. In the past 10 years, researchers have been trying to reduce tumor radiation resistance by improving tumor blood supply. Pre-clinical models have shown that antiangiogenesis therapy can temporarily "normalize" the tumor vasculature, allowing it to supply oxygen more effectively, and increasing its sensitivity to radiotherapy (13). Endostar is a broad-spectrum angiogenesis inhibitor, which is known to inhibit the signal pathway through vascular endothelial growth factor (VEGF) and fibroblast growth factor (FGF) to regulate the survival, proliferation, and migration of endothelial cells (14).

After a series of clinical studies, Yang et al. (15-18) determined the dosage and mode of administration of Endostar combined with chemotherapy in the treatment of advanced NSCLC. However, the same mode of administration combined with CCRT did not show an improvement in efficacy in the treatment of locally advanced NSCLC, while toxic side effects were increased (19). Taken that mode of administration of Endostar combined with radiotherapy may affect the efficacy and toxic side effects in the treatment of locally advanced NSCLC, our research team conducted an animal study, which showed that the tumor blood vessels were normalized after 5-7 days of treatment with Endostar, the hypoxic environment was improved, and the best tumor suppression effects were achieved under combination with radiotherapy $(20,21)$. Based on in vitro evidences, a Phase II clinical study was designed to explore the optimal mode of the combination of fortnightly-administered Endostar and CCRT in the treatment of inoperable locally advanced NSCLC, and the initial therapeutic effects were satisfactory $(22,23)$.

In this study, long-term follow-up visits were paid to participants in pre-liminary studies, and the data was compared with patients who received only CCRT during the same time. The objective aimed to evaluate the mechanisms of how anti-angiogenesis therapy can temporarily "normalize" the tumor vasculature, as well as whether the fortnightly-administration of Endostar combined with CCRT can improve the 5-year survival rate for patients with locally advanced NSCLC. We present the following article in accordance with the STROBE reporting checklist (available at https://dx.doi.org/10.21037/apm-21-1092).

\section{Methods}

\section{The sources of the patients and the inclusion criteria}

From 2009 to 2015, our research team carried out two prospective phase II clinical studies (ClinicalTrials.gov, No. NCT01218594 and No. NCT01733589), using fortnightly administration of Endostar combined with CCRT to treat locally advanced NSCLC patients (CCRT + Endostar group). A total of 227 patients with treatment-naive inoperable locally advanced NSCLC who had the data of long-term follow-up visits in the same period were included retrospectively, and received radical CCRT (CCRT group). Three-dimensional conformal radiation therapy (3D-CRT) or intensity-modulated radiation therapy (IMRT) was applied in both groups, and the regimens of both groups were platinum-based 2-drug regimens. Excluding patients with a radiotherapy dose of $<60 \mathrm{~Gy}, 193$ patients were finally included in the analysis, with 104 cases in the CCRT + Endostar group and 89 cases in the CCRT group. The patients in this study was strictly included according to the inclusion and exclusion criteria from two clinical studies (ClinicalTrials.gov, No. NCT01218594 and No. NCT01733589) to achieve a relatively balanced exposure groups at baseline was used in this study to reduce selective bias. All procedures performed in this study involving human participants were in accordance with the Declaration of Helsinki (as revised in 2013). The study was approved by ethics committee of The Cancer Hospital of the University of Chinese Academy of Sciences (Zhejiang Cancer Hospital) (No. ZJZLYY-2012-10-24). Individual consent for this retrospective analysis was waived. 


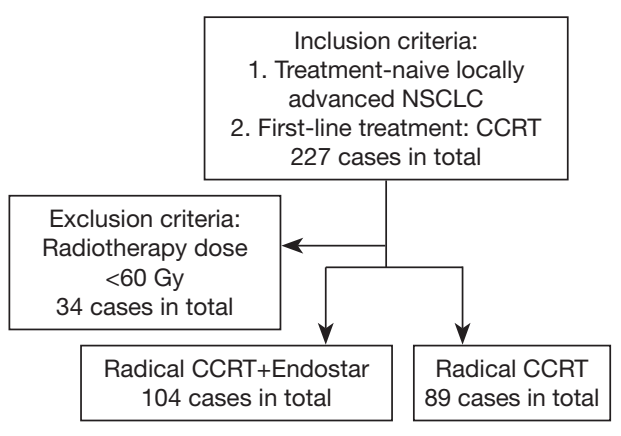

Figure 1 STROBE diagram of flowchart of patient inclusion. NSCLC, non-small cell lung cancer; CCRT, concurrent chemoradiotherapy.

\section{Treatment}

\section{Radiotherapy}

Either 3D-CRT or IMRT was applied in both groups, and the dose of radical radiotherapy was given, conventional fractionated $2 \mathrm{~Gy} / \mathrm{F}$, once a day, 5 times a week.

\section{Chemotherapy}

Participants received concurrent platinum-based 2-drug CCRT, including EP regimen (etoposide $50 \mathrm{mg} / \mathrm{m}^{2}$, D1-D5; cisplatin $50 \mathrm{mg} / \mathrm{m}^{2}, \mathrm{D} 1$ and D8, repeated every 4 weeks), DP/C regimen [docetaxel $65 \mathrm{mg} / \mathrm{m}^{2}$, D1; cisplatin $65 \mathrm{mg} / \mathrm{m}^{2}$ or carboplatin area under the curve $(\mathrm{AUC})=5$, D1, repeated every 4 weeks], or TP/C regimen (paclitaxel $135-175 \mathrm{mg} / \mathrm{m}^{2}$, D1; cisplatin $65 \mathrm{mg} / \mathrm{m}^{2}$ or carboplatin AUC $=5, \mathrm{D} 1$, repeated every 4 weeks), NP regimen (vinorelbine $60 \mathrm{mg} / \mathrm{m}^{2}, \mathrm{D} 1$ and D8; cisplatin $65 \mathrm{mg} / \mathrm{m}^{2}, \mathrm{D} 1$, repeated every 4 weeks), AP regimen (pemetrexed $500 \mathrm{mg} / \mathrm{m}^{2}$, D1; cisplatin $65 \mathrm{mg} / \mathrm{m}^{2}$, D1, repeated every 4 weeks), and GP regimen (gemcitabine $1,000 \mathrm{mg} / \mathrm{m}^{2}, \mathrm{D} 1$ and D8; cisplatin $65 \mathrm{mg} / \mathrm{m}^{2}$, D1, repeated every 4 weeks).

\section{Endostar}

In the CCRT + Endostar group, the dose of Endostar was $7.5 \mathrm{mg} / \mathrm{m}^{2}$ per day with $5-7$ days of continuous administration. The administration of Endostar was given 5-7 days before radiotherapy, and fortnightly during radiotherapy. The cycle was repeated every 2 weeks to a total of 4 cycles.

\section{Statistical analysis}

The OS calculation was as follows: the duration from the start of treatment to death due to any cause. In the case of survival, the OS was recorded to the time point of the last follow-up visit. Local progression-free survival (LPFS) was calculated as follows: duration from the start of treatment to local tumor progression, including the relapse time of the primary lesion and regional lymph nodes (including relapse both in- and out of the irradiation field). Distant metastasisfree survival (DMFS) was calculated as follows: duration from the start of treatment to the first appearance of distant metastasis. The software SPSS 24.0 (IBM Inc., Armonk, NY, USA) was employed for statistical analysis. The chisquare test was used to compare participant characteristics and treatment-related factors. The Kaplan-Meier estimator was used for survival analysis, and log-rank test was used for OS inter-group comparison. The hazard ratio (HR) and 95\% confidence interval (95\% CI) for Cox-model were used to perform univariate survival analysis for variables which may have affected the results, and the Cox proportionalhazards regression model was used for multivariate survival analysis for statistically significant univariate factors. All statistical tests used a 2-sided hypothesis test with type I error. $\mathrm{P}<0.05$ was deemed statistically significant.

\section{Results}

\section{The comparison of baseline characteristics between two groups}

Two hundred twenty seven patients with treatmentnaive inoperable locally advanced NSCLC from two prospective phase II clinical studies (ClinicalTrials.gov, No. NCT01218594 and No. NCT01733589) conducted from 2009 to 2015 were screened and among them, 34 patients were excluded due to Radiotherapy dose of $<60 \mathrm{~Gy}$. A total of 193 patients were enrolled in this study, including 104 patients in the CCRT + Endostar group and 89 patients in the CCRT group (Figure 1). Baseline characteristics of the Endostar + CCRT and CCRT groups were as follows: median age: 58 and 56 years, respectively; there were $88(84.6 \%)$ and $74(83.1 \%)$ male participants, and $16(15.4 \%)$ and $15(16.9 \%)$ females, respectively. There were 63 cases $(60.6 \%)$ and 40 cases $(44.9 \%)$ of squamous cell carcinoma, respectively. The stage IIIA and stage IIIB were $33.7 \%$ and $66.3 \%$ in CCRT + Endostar group and $28.1 \%$ and $71.9 \%$ in CCRT group. The regimens of CCRT in the CCRT + Endostar group were EP regimen (61.5\%) and DP regimen (38.5\%), while a variety of platinum-based 2-drug chemotherapy regimens were used in the CCRT group, with the TP/C regimen in the majority (53.9\%), 20 cases of DP regimen 
(22.5\%), 3 cases of EP regimen (3.4\%), and 18 cases (20.2\%) of other regimens. Baseline characteristics and treatment condition of participants in two groups were displayed, and the two groups of patients were comparable in terms of baseline demographics and clinical data in Table 1 .

\section{Survival analysis}

The median follow-up times of the CCRT + Endostar group and CCRT group were 73.6 months (95\% CI: 65.6 to 81.7 months) and 66.3 months (95\% CI: 52.7 to 79.9 months), respectively. A total of 72 participants died in the CCRT + Endostar group, of which 64 cases died of lung cancer progression, 5 died of other diseases, 2 died of treatment complications, and 1 case died of unknown cause; in the CCRT group 62 participants died, of which 60 cases died of lung cancer progression, and 2 of other diseases. The median survival times of two groups were 29.7 months (95\% CI: 22.8 to 36.6 months) and 21.3 months (95\% CI: 15.9 to 26.7 months), the 5 -year survival rates were $34.7 \%$ and $23.6 \%$, respectively $(\mathrm{P}=0.038)$. The $\mathrm{OS}$ curves were shown in Figure 2. According to the univariate analysis, the differences in the effects of gender, age stratification, Eastern Cooperative Oncology Group (ECOG) score, pathological type, Union for International Cancer Control (UICC) staging, radiotherapy technology, and whether EP regimen was used in concurrent chemotherapy on OS were statistically significant (Table 2).

\section{Late-stage adverse reactions}

The records of late-stage adverse reactions mainly included late-stage lung injury and esophageal injury. Late-stage lung injury and esophageal injury after radiotherapy were evaluated according to standards of the Radiation Therapy Oncology Group (RTOG) and the European Organization for Research and Treatment of Cancer (EORTC). No grade 4-5 late-stage lung injury occurred in the CCRT + Endostar group or CCRT group. A single case of grade 3 lung injury was observed in the CCRT + Endostar group. The incidence rates of grade 1 and grade 2 lung injury in the CCRT group were significantly higher than that in the CCRT + Endostar group, which were $33.7 \%$ and $14.4 \%$, $9.1 \%$ and $3.8 \%$, respectively $(\mathrm{P}=0.002)$. The incidence rate of late-stage esophageal injury was low in both groups, which were $5.6 \%$ and $1.0 \%$ respectively $(\mathrm{P}=0.188)$. The late-stage adverse reactions are shown in Table 3.

\section{Discussion}

In this study, long-term follow-up visits were paid to participants in pre-clinical studies, and the data were retrospectively compared with those who received only CCRT at the same time, elucidating the mechanisms of how anti-angiogenesis therapy can temporarily "normalize" the tumor vasculature, and that fortnightly administration of Endostar combined with CCRT can improve the 5-year survival rate for patients with locally advanced NSCLC. In this study, the median follow-up time of the CCRT + Endostar group and CCRT group was 73.6 months (95\% CI: 65.6 to 81.7 months) and 66.3 months (95\% CI: 52.7 to 79.9 months), respectively. The median follow-up time of the two groups both exceeded 5 years. The median OS and 5-year OS rate of the CCRT + Endostar group were significantly better than those of the CCRT group (29.7 vs. 21.3 months, $34.7 \%$ vs. $23.6 \%$, respectively, $\mathrm{P}=0.038$ ).

In the study of locally advanced NSCLC with longer follow-up period, the median follow-up time reported by Southwest Oncology Group (SWOG) 9019 was 52 months, the median OS was 15 months, and the 3 - and 5 -year OS rates were $17 \%$ and $15 \%$, respectively (3). In a study using cetuximab combined with CCRT to treat patients with stage III NSCLC, the median follow-up time was 32 months, and the median OS was 25.2 months (5). The RTOG 0617 (2) study compared the efficacy of CCRT with different radiotherapy doses of 60 and 74 Gy in the treatment of locally advanced NSCLC. The median followup time was up to 5.1 years, median survival time was 28.7 and 20.3 months, and the 5 -year OS rate was $32.1 \%$ and $23 \%$, respectively. In that study, $69 \%$ of patients had stageIIIA disease; while in this study participants with stage-IIIB disease accounted for $66.3 \%$ and $71.9 \%$ in two groups. The OS of the CCRT group was not as good as the that of the 60 Gy group in the RTOG 0617 study, but the outcome of participants in the CCRT + Endostar group was comparable to that of the 60 Gy group in the RTOG 0617 study (median OS was 29.7 and 28.7 , and 5 -year OS rate was $34.7 \%$ and $32.1 \%$, respectively).

The PACIFIC study (10) had achieved great success as it conducted durvalumab consolidation immunotherapy after CCRT in the treatment of inoperable locally advanced NSCLC. Survival data were updated in 2017 (9): the median follow-up time was 25.2 months, median OS was not reached (95\% CI: 34.7 to NR), which was significantly 
Table 1 Baseline characteristics and treatment conditions in two groups

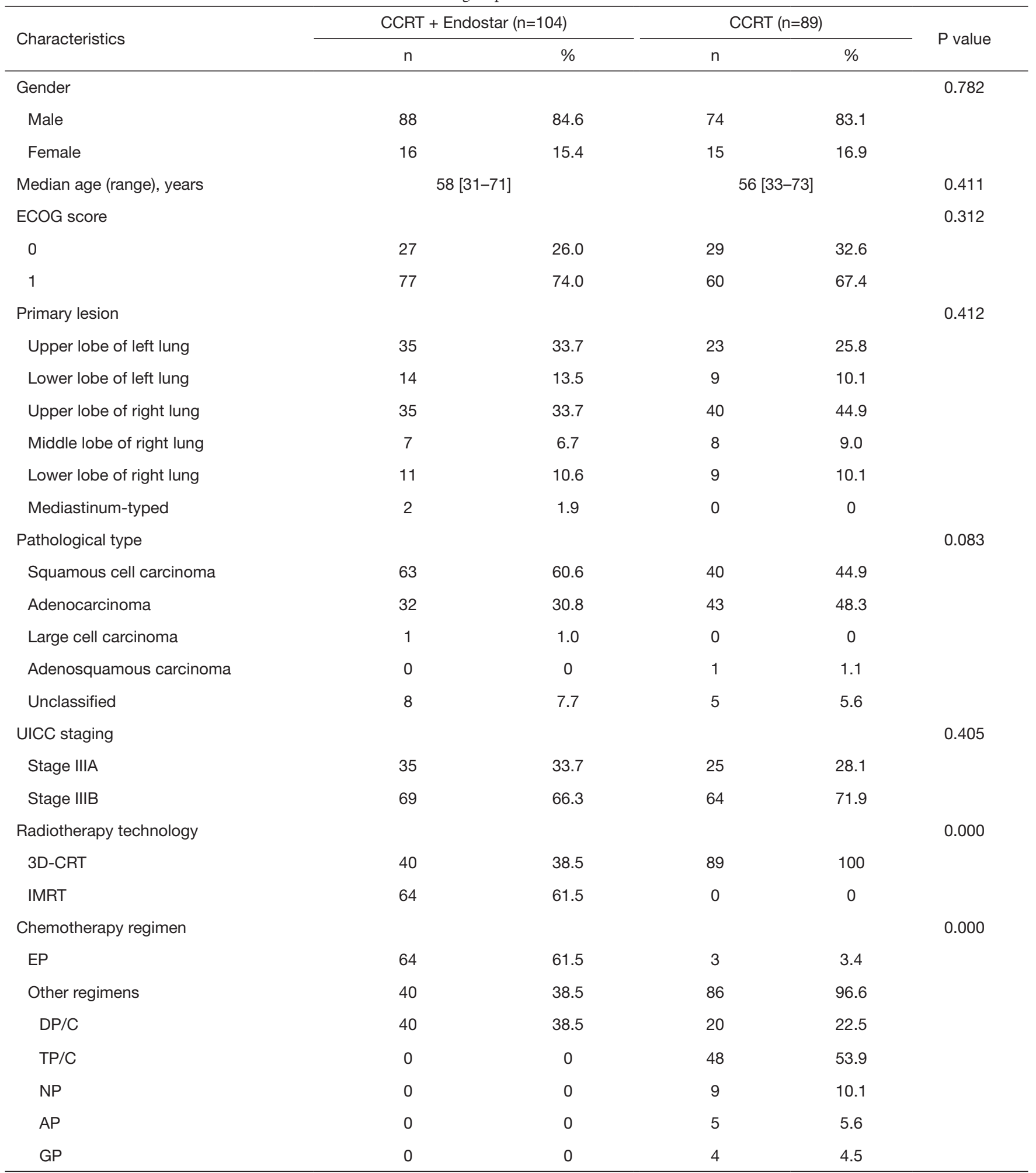

EP, etoposide, cisplatin; DP/C, docetaxel, cisplatin or carboplatin; TP/C, paclitaxel, cisplatin or carboplatin; NP, vinorelbine, cisplatin; AP, pemetrexed, cisplatin; GP, gemcitabine, cisplatin; ECOG, Eastern Cooperative Oncology Group; UICC, Union for International Caner Control; 3D-CRT, three-dimensional conformal radiation therapy. 


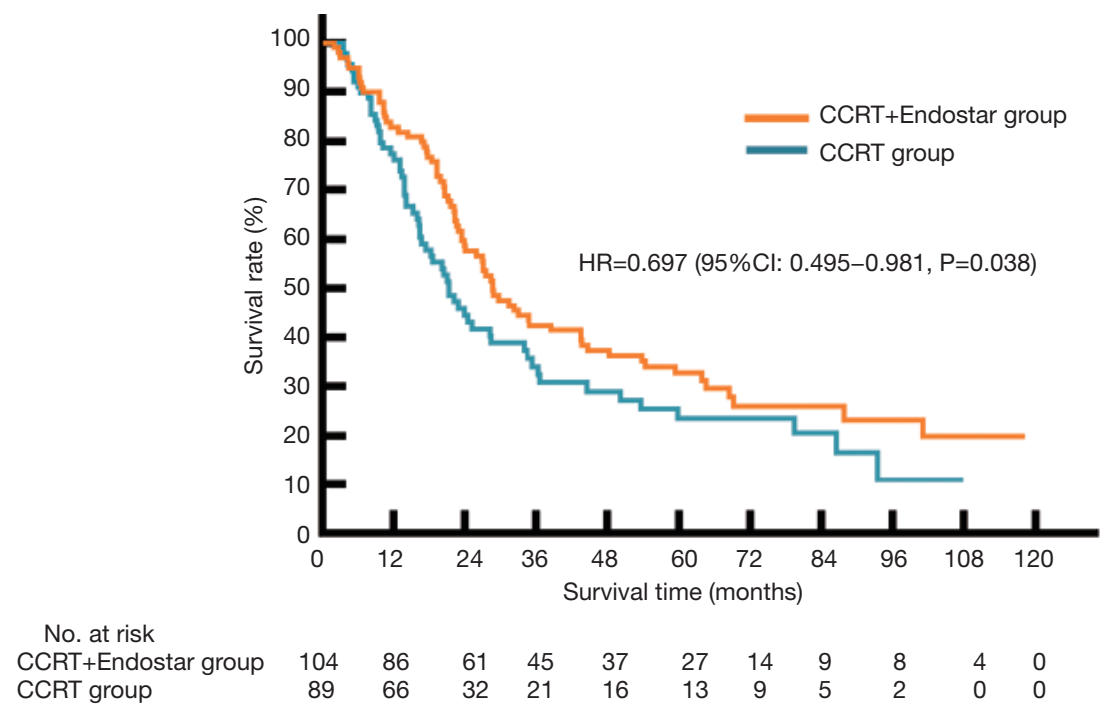

Figure 2 The OS curves of two groups. CCRT, concurrent chemoradiotherapy; OS, overall survival.

Table 2 Univariate analysis

\begin{tabular}{llc}
\hline Factors & $\mathrm{HR}(95 \% \mathrm{Cl})$ & $\mathrm{P}$ value \\
\hline Treatment plan (CCRT + Endostar/CCRT) & $0.697(0.495-0.981)$ & 0.038 \\
Gender (male/female) & $1.222(0.766-1.949)$ & 0.400 \\
Age stratification (<60/ $\geq 60$ years old) & $0.855(0.605-1.207)$ & 0.372 \\
ECOG score (0/1) & $0.889(0.603-1.309)$ & 0.550 \\
Pathological type (squamous cell carcinoma/non-squamous cell carcinoma) & $1.044(0.652-1.673)$ & 0.858 \\
UICC staging (IIIA/IIIB) & $0.868(0.602-1.252)$ & 0.449 \\
Radiotherapy technology (3D-CRT/IMRT) & $1.304(0.900-1.889)$ & 0.160 \\
Chemotherapy regimen (EP regimen/non-EP regimen) & $0.869(0.604-1.250)$ & 0.449 \\
\hline
\end{tabular}

CCRT, concurrent chemoradiotherapy; HR, hazard ratio; CI, confidence interval; ECOG, Eastern Cooperative Oncology Group; UICC, Union for International Caner Control; 3D-CRT/IMRT, three-dimensional conformal radiation therapy or intensity-modulated radiation therapy; EP, etoposide, cisplatin.

superior to that of the CCRT group (28.7 months, 95\% CI: 22.9 to NR), $(\mathrm{P}=0.0025)$. For this reason, durvalumab consolidation immunotherapy after CCRT has become the new standard treatment for locally advanced NSCLC in 2018. Based on this, we did not further carry out a prospective RCT to compare the combination of Endostar and CCRT with CCRT alone. However, the PACIFIC study conducted a high-standard screening of its patients. The participants were those who had no disease progression after CCRT, and some who had shown resistance to CCRT were dropped. Therefore, challenges remain in how to overcome the resistance of patients with lung cancer to chemoradiotherapy and improve the efficacy of CCRT.

Increasingly, studies have also shown that appropriate anti-angiogenic drugs can promote the normalization of tumor blood vessels within a certain time window, which can directly alleviate hypoxia and make the tumor immune environment change from an immunosuppressive state to an immune-supportive one (24). The mechanism is mainly manifested in the following aspects (25): (I) promote $\mathrm{T}$ cell infiltration; (II) reduce hypoxia, preferentially induce the polarization of tumor-associated macrophages (TAMs) to M1 phenotype; (III) blood vessel normalization reduces the recruitments of regulatory $\mathrm{T}$ cells (Treg) and myeloid- 
Table 3 The late-stage adverse reactions in two groups

\begin{tabular}{|c|c|c|c|c|c|}
\hline \multirow{2}{*}{$\begin{array}{l}\text { Late-stage adverse } \\
\text { reactions }\end{array}$} & \multicolumn{2}{|c|}{$\begin{array}{c}\text { CCRT + Endostar } \\
(n=104)\end{array}$} & \multicolumn{2}{|c|}{ CCRT $(n=89)$} & \multirow[t]{2}{*}{$P$ value } \\
\hline & $\mathrm{n}$ & $\%$ & $\mathrm{n}$ & $\%$ & \\
\hline \multicolumn{3}{|l|}{ Late-stage lung injury } & & & 0.002 \\
\hline Grade 1 & 15 & 14.4 & 30 & 33.7 & \\
\hline Grade 2 & 4 & 3.8 & 8 & 9.10 & \\
\hline Grade 3 & 1 & 1.0 & 0 & 0 & \\
\hline Total & 20 & 19.2 & 38 & 42.8 & \\
\hline \multicolumn{3}{|c|}{ Late-stage esophageal injury } & & & 0.188 \\
\hline Grade 1 & 0 & 0 & 1 & 1.1 & \\
\hline Grade 2 & 0 & 0 & 3 & 3.4 & \\
\hline Grade 3 & 1 & 1.0 & 1 & 1.1 & \\
\hline Total & 1 & 1.0 & 5 & 5.6 & \\
\hline
\end{tabular}

CCRT, concurrent chemoradiotherapy.

derived suppressor cells (MDSC); (IV) block the inhibiting signals of the differentiation of dendritic cells (DC); (V) immunosuppressive signals such as programmed deathligand 1 (PD-L1) induced by hypoxia can be down-regulated by improving perfusion. Various mechanisms suggest that anti-tumor angiogenesis therapy and immunotherapy can promote compensate for each other, achieving a synergistic interaction. At present, many ongoing studies to explore the combination of anti-angiogenesis therapy and immunotherapy have been registered in ClinicalTrials.gov.

The combination of anti-angiogenic drugs and immunotherapy has become a new direction of exploration; concurrently, the toxic and side effects have also become a special concern of researchers. Endostar showed a better safety profile than other antiangiogenic drugs, and the side effect of Endostar less than that of other antiangiogenic drugs might be related to its own structure and biological characteristics. Recombinant human endostatin (rhE, Endostar), a C-terminal fragment naturally derived from type XVIII collagen could maintain good structural stability and specifically inhibit the activity of vascular endothelial growth factor to block angiogenesis as well as induce cancer cell apoptosis, which could better simulate the roles of endostatin in tumor suppression in vivo than other antiangiogenic drugs with less side effect (23). However, more researches and clinical trials remained to be conducted for further verification of the link between less side effect of Endostar and its lower dosage.
Among the currently available anti-angiogenic drugs, Endostar, bevacizumab and Anlotinib were the most applied in the treatment of various cancer types. Based on previously reported evidences, the advantages of Endostar over two other antiangiogenic drugs could summarized as follows, better suitability for treatment of more extensive pathologic types, wider patient coverage than other antiangiogenic drugs, a better safety profile and less toxic side effects, capability of being used in combination with radiotherapy $(23,26-30)$. Among other anti-angiogenic drugs, the reported toxic and side effects mainly include vascular events, cardiovascular toxicity, proteinuria, delayed wound healing, intestinal perforation, and esophagotracheal fistula. A phase II RCT of bevacizumab combined with carboplatin and paclitaxel in the first-line treatment of advanced NSCLC showed that the increase in pulmonary hemorrhage was related to the mass or cavity of the central part, and $4 / 6$ of the patients had squamous cell carcinoma. The risk of pulmonary hemorrhage for squamous cell carcinoma was $31 \%$, and the risk of pulmonary hemorrhage for other pathological subtypes was $4 \%$ (31). Therefore, in the subsequent phase III clinical study (ECOG 4599), the efficacy of paclitaxel plus carboplatin combined with bevacizumab in the treatment of recurrent or advanced NSCLC was evaluated and compared with the efficacy of the chemotherapy. Patients with squamous cell carcinoma and hemoptysis were excluded (32). The SWOG S0533 study (33) aimed to evaluate the feasibility of using bevacizumab combined with CCRT for stage III NSCLC. The study was terminated due to the occurrence of severe esophagotracheal fistula. The Food and Drug Administration (FDA) also issued a warning: there is a risk of esophagotracheal fistula when lung cancer patients receive bevacizumab combined with chemoradiotherapy, and the corresponding warnings should be made clear on the drug insert. Therefore, in the combination use of anti-angiogenic drugs and radiotherapy, clinical studies were difficult to be carried out due to the concerns about fistulas, hemorrhage, and other serious toxic and side effects, especially in patients with squamous cell carcinoma. At present, tumor vascular inhibitors used in China include anlotinib and apatinib, both of which were used for the monotherapy or the combination with chemotherapy in the second-line and third-line treatment studies, and there is a lack of safety data for their combination with radiotherapy.

Endostar is administered fortnightly, and it was shown to be tolerated well in the prospective phase II clinical study of 
our research team. Except for 1 case of grade 5 hemoptysis due to the great vessels involved by the tumor, no serious anti-angiogenesis drugs-related bleeding adverse reactions occurred. The most common acute toxicity can be attributed to the toxic and side effects of chemoradiotherapy, including blood toxicity, esophagitis, and pneumonia. The incidence rate was lower than or similar to the results reported in previous studies using CCRT for NSCLC $(5,34,35)$. With the advent of the age of immunotherapy, the possible lung injury caused by the combination use of chest radiotherapy and immunotherapy has also become the special concern of researchers. In our phase II study of the combination use of Endostar and CCRT for locally advanced NSCLC, the incidence rate of grade $3-5$ acute radiation pneumonitis was $6.8 \%$, which was still lower than the that reported by Cancer and Leukemia Group B (CALGB) 30407 (12\%) (5). Due to the poor therapeutic effects on lung cancer and short survival period, reports of late-stage radiation injury were rarely seen. In this study, except for 1 case of grade 3 late-stage lung injury in the CCRT + Endostar group, the lung injuries were mainly grade 1-2 in two groups. The incidence rate of CCRT + Endostar group was significantly lower than that of the CCRT group (19.2\% vs. $42.8 \%$, respectively). In this study, 4 participants in the CCRT group received GP regimen in concurrent chemotherapy despite studies having shown that the combined use of radiotherapy and concurrent GP regimen (gemcitabine + cisplatin) can increase the incidence rate of symptomatic radiation pneumonitis (36), in which 2 patients developed grade 1 late-stage lung injury, 1 developed grade 2 lung injury, no patient developed $\geq$ grade 3 lung injury, and the incidence rate of late-stage radiation-induced lung injury was not higher than other chemotherapy regimens (Table S1). The pathological features of radiation-induced lung injury mainly include inflammatory cell infiltration, type I alveolar cell injury, increased capillary permeability, alveolar interstitial edema, and thickened alveolar septum, resulting in the occlusion of residual alveoli and capillary cavities, and finally leading to pulmonary fibrosis. From the perspective of pathophysiology, a series of proinflammatory factors and growth factors are involved in the process of radiation-induced lung injury (37). Jackson et al. (38) demonstrated that radiation damage was related to the increase of macrophage infiltration induced by hypoxia and the production of transforming growth factor beta (TGF- $\beta$ ) and VEGF. According to a report by Tanabe et al. (39), endostatin can significantly inhibit the expression of VEGF-A, alpha-smooth muscle actin ( $\alpha$-SMA) and the pro-fibrotic factor TGF- $\beta 1$ in a mouse model, and inhibit peritoneal fibrosis in a dose-dependent manner. An experimental study found that compared with the animals exposed to radiation but not treated with Endostar, those animals treated with Endostar were relatively mild in pulmonary alveolitis, pulmonary edema, and fibrosis degree, and lower in TGF- $\beta 1$ expression, suggesting that Endostar can relieve the degree of radiation-induced pulmonary lesions (40). In this study, the CCRT + Endostar group adopted a fortnightly-administration mode, using antiangiogenic drugs to "normalize" tumor blood vessels in the window period and combining with chemoradiotherapy. On the one hand, previous reports revealed that Endostar increased the safety and efficacy of chemoradiotherapy in several cancers $(41,42)$. On the other hand, pre-clinical results showed that Endostar may reduce the incidence rates of acute radiation-induced lung injury (RILI) by inhibiting the expression of inflammatory factors related to RILI (40). Besides, previous study revealed that Endostar was involved in the regulation of both metabolism and tumor microenvironment hypoxia, which may be responsible for enhanced antitumor effects of Endostar combined with radiotherapy (43). For inoperable locally advanced NSCLC, further exploration and research into whether the addition of Endostar on the basis of the new standard of CCRT plus consolidation immunotherapy can further improve the therapeutic effects and also play a role in radiological protection is warranted.

The limitations in this study should never be neglected. For one thing, this was a retrospective study with a small sample size, and prospective clinical randomized controlled trials (RCTs) will be conducted in the future for further verification of the efficacy and safety of Endostar combined with CCRT in the treatment of inoperable locally advanced non-small cell lung cancer. For another, heterogeneity of patients enrolled in clinical trials should be further considered in the future to reduce potential selective bias. Furthermore, the optimal dosage, frequency and course of treatment of Endostar in combination with CCRT in the treatment of inoperable locally advanced non-small cell lung cancer should be further investigated and explored for better validation.

\section{Conclusions}

The combined use of radical CCRT and fortnightlyadministered endostatin (Endostar) significantly improved the median OS and 5-year survival rate for patients with 
inoperable locally advanced NSCLC, and the toxic and side effects were significantly lower than those of patients received CCRT. In the age of immunotherapy, CCRT plus consolidation immunotherapy has become the new treatment standard for locally advanced NSCLC. The effectiveness and safety of anti-angiogenic drugs, immunotherapy, and CCRT in the treatment of inoperable locally advanced NSCLC should be further explored.

\section{Acknowledgments}

Funding: Zhejiang Provincial Natural Science Foundation (No. LZ13H160003).

\section{Footnote}

Reporting Checklist: The authors have completed the STROBE reporting checklist. Available at https://dx.doi. org/10.21037/apm-21-1092

Data Sharing Statement: Available at https://dx.doi. org/10.21037/apm-21-1092

Conflicts of Interest: All authors have completed the ICMJE uniform disclosure form (available at https://dx.doi. org/10.21037/apm-21-1092). The authors have no conflicts of interest to declare.

Ethical Statement: The authors are accountable for all aspects of the work in ensuring that questions related to the accuracy or integrity of any part of the work are appropriately investigated and resolved. All procedures performed in this study involving human participants were in accordance with the Declaration of Helsinki (as revised in 2013). The study was approved by ethics committee of The Cancer Hospital of the University of Chinese Academy of Sciences (Zhejiang Cancer Hospital) (No. ZJZLYY-2012-10-24). Individual consent for this retrospective analysis was waived.

Open Access Statement: This is an Open Access article distributed in accordance with the Creative Commons Attribution-NonCommercial-NoDerivs 4.0 International License (CC BY-NC-ND 4.0), which permits the noncommercial replication and distribution of the article with the strict proviso that no changes or edits are made and the original work is properly cited (including links to both the formal publication through the relevant DOI and the license). See: https://creativecommons.org/licenses/by-nc-nd/4.0/.

\section{References}

1. Aupérin A, Le Péchoux C, Rolland E, et al. Meta-analysis of concomitant versus sequential radiochemotherapy in locally advanced non-small-cell lung cancer. J Clin Oncol 2010;28:2181-90.

2. Bradley JD, Hu C, Komaki RR, et al. Long-Term Results of NRG Oncology RTOG 0617: Standard- Versus HighDose Chemoradiotherapy With or Without Cetuximab for Unresectable Stage III Non-Small-Cell Lung Cancer. J Clin Oncol 2020;38:706-14.

3. Zhou Q, Chen M, Wu G, et al. GEMSTONE-301: a phase III clinical trial of CS1001 as consolidation therapy in patients with locally advanced/unresectable (stage III) non-small cell lung cancer (NSCLC) who did not have disease progression after prior concurrent/sequential chemoradiotherapy. Transl Lung Cancer Res 2020;9:2008-15.

4. Bradley JD, Paulus R, Komaki R, et al. Standard-dose versus high-dose conformal radiotherapy with concurrent and consolidation carboplatin plus paclitaxel with or without cetuximab for patients with stage IIIA or IIIB non-small-cell lung cancer (RTOG 0617): a randomised, two-by-two factorial phase 3 study. Lancet Oncol 2015;16:187-99.

5. $\mathrm{Hu} M, \mathrm{Xu} \mathrm{Q}$, Yang S, et al. Pretreatment systemic inflammation response index (SIRI) is an independent predictor of survival in unresectable stage III non-small cell lung cancer treated with chemoradiotherapy: a twocenter retrospective study. Ann Transl Med 2020;8:1310.

6. Hanna N, Neubauer M, Yiannoutsos C, et al. Phase III study of cisplatin, etoposide, and concurrent chest radiation with or without consolidation docetaxel in patients with inoperable stage III non-small-cell lung cancer: the Hoosier Oncology Group and U.S. Oncology. J Clin Oncol 2008;26:5755-60.

7. Zou L, Chu L, Xia F, et al. Is clinical target volume necessary? - a failure pattern analysis in patients with locally advanced non-small cell lung cancer treated with concurrent chemoradiotherapy using intensity-modulated radiotherapy technique. Transl Lung Cancer Res 2020;9:1986-95.

8. Vokes EE, Herndon JE 2nd, Kelley MJ, et al. Induction chemotherapy followed by chemoradiotherapy compared with chemoradiotherapy alone for regionally advanced unresectable stage III Non-small-cell lung cancer: Cancer and Leukemia Group B. J Clin Oncol 2007;25:1698-704.

9. Antonia SJ, Villegas A, Daniel D, et al. Overall Survival 
with Durvalumab after Chemoradiotherapy in Stage III NSCLC. N Engl J Med 2018;379:2342-50.

10. Antonia SJ, Villegas A, Daniel D, et al. Durvalumab after Chemoradiotherapy in Stage III Non-Small-Cell Lung Cancer. N Engl J Med 2017;377:1919-29.

11. Carmeliet P, Jain RK. Principles and mechanisms of vessel normalization for cancer and other angiogenic diseases. Nat Rev Drug Discov 2011;10:417-27.

12. Vaupel P, Mayer A. Hypoxia in cancer: significance and impact on clinical outcome. Cancer Metastasis Rev 2007;26:225-39.

13. Jain RK. Normalization of tumor vasculature: an emerging concept in antiangiogenic therapy. Science 2005;307:58-62.

14. Abdollahi A, Hahnfeldt P, Maercker C, et al. Endostatin's antiangiogenic signaling network. Mol Cell 2004;13:649-63.

15. Wang J, Sun Y, Liu Y, et al. Results of randomized, multicenter, double-blind phase III trial of rh-endostatin (YH-16) in treatment of advanced non-small cell lung cancer patients. Zhongguo Fei Ai Za Zhi 2005;8:283-90.

16. Yang L, Wang JW, Cui CX, et al. Rh-endostatin (YH-16) in combination with vinorelbine and cisplatin for advanced non-small cell lung cancer:a multicenter phase II trial. Chinese New Drugs Journal 2005;14:204-7.

17. Yang L, Wang JW, Sun Y, et al. Randomized phase II trial on escalated doses of Rh-endostatin (YH-16) for advanced non-small cell lung cancer. Zhonghua Zhong Liu Za Zhi 2006;28:138-41.

18. Yang L, Wang JW, Tang ZM, et al. A phase I clinical trial for recombinant human endostatin. Chinese New Drugs Journal 2004;13:548-53.

19. Sun XJ, Deng QH, Yu XM, et al. A phase II study of Endostatin in combination with paclitaxel, carboplatin, and radiotherapy in patients with unresectable locally advanced non-small cell lung cancer. BMC Cancer 2016;16:266.

20. Peng F, Xu Z, Wang J, et al. Recombinant human endostatin normalizes tumor vasculature and enhances radiation response in xenografted human nasopharyngeal carcinoma models. PLoS One 2012;7:e34646.

21. Peng F, Wang J, Zou Y, et al. Recombinant human endostatin improves tumor vasculature and alleviates hypoxia in Lewis lung carcinoma. Chin J Radiat Oncol 2011;20:69-72.

22. Bao Y, Peng F, Zhou QC, et al. Phase II trial of recombinant human endostatin in combination with concurrent chemoradiotherapy in patients with stage III non-small-cell lung cancer. Radiother Oncol 2015;114:161-6.
23. Zhai Y, Ma H, Hui Z, et al. HELPER study: A phase II trial of continuous infusion of endostar combined with concurrent etoposide plus cisplatin and radiotherapy for treatment of unresectable stage III non-small-cell lung cancer. Radiother Oncol 2019;131:27-34.

24. Huang Y, Yuan J, Righi E, et al. Vascular normalizing doses of antiangiogenic treatment reprogram the immunosuppressive tumor microenvironment and enhance immunotherapy. Proc Natl Acad Sci U S A 2012;109:17561-6.

25. Yi M, Jiao D, Qin S, et al. Synergistic effect of immune checkpoint blockade and anti-angiogenesis in cancer treatment. Mol Cancer 2019;18:60.

26. Lind JS, Senan S, Smit EF. Pulmonary toxicity after bevacizumab and concurrent thoracic radiotherapy observed in a phase I study for inoperable stage III nonsmall-cell lung cancer. J Clin Oncol 2012;30:e104-8.

27. Han B, Li K, Wang Q, et al. Effect of Anlotinib as a Third-Line or Further Treatment on Overall Survival of Patients With Advanced Non-Small Cell Lung Cancer: The ALTER 0303 Phase 3 Randomized Clinical Trial. JAMA Oncol 2018;4:1569-75.

28. Jiang X, Ding M, Qiao Y, et al. Recombinant human endostatin combined with radiotherapy in the treatment of brain metastases of non-small cell lung cancer. Clin Transl Oncol 2014;16:630-6.

29. Niho S, Kunitoh H, Nokihara H, et al. Randomized phase II study of first-line carboplatin-paclitaxel with or without bevacizumab in Japanese patients with advanced non-squamous non-small-cell lung cancer. Lung Cancer 2012;76:362-7.

30. Spigel DR, Hainsworth JD, Yardley DA, et al. Tracheoesophageal fistula formation in patients with lung cancer treated with chemoradiation and bevacizumab. J Clin Oncol 2010;28:43-8.

31. Johnson DH, Fehrenbacher L, Novotny WF, et al. Randomized phase II trial comparing bevacizumab plus carboplatin and paclitaxel with carboplatin and paclitaxel alone in previously untreated locally advanced or metastatic non-small-cell lung cancer. J Clin Oncol 2004;22:2184-91.

32. Sandler A, Gray R, Perry MC, et al. Paclitaxel-carboplatin alone or with bevacizumab for non-small-cell lung cancer. N Engl J Med 2006;355:2542-50.

33. Wozniak AJ, Moon J, Thomas CR Jr, et al. A Pilot Trial of Cisplatin/Etoposide/Radiotherapy Followed by Consolidation Docetaxel and the Combination of Bevacizumab (NSC-704865) in Patients With Inoperable 
Locally Advanced Stage III Non-Small-Cell Lung Cancer: SWOG S0533. Clin Lung Cancer 2015;16:340-7.

34. Blumenschein GR Jr, Paulus R, Curran WJ, et al. Phase II study of cetuximab in combination with chemoradiation in patients with stage IIIA/B non-small-cell lung cancer: RTOG 0324. J Clin Oncol 2011;29:2312-8.

35. Curran WJ Jr, Paulus R, Langer CJ, et al. Sequential vs. concurrent chemoradiation for stage III non-small cell lung cancer: randomized phase III trial RTOG 9410. J Natl Cancer Inst 2011;103:1452-60.

36. Liu T, He Z, Dang J, et al. Comparative efficacy and safety for different chemotherapy regimens used concurrently with thoracic radiation for locally advanced non-small cell lung cancer: a systematic review and network metaanalysis. Radiat Oncol 2019;14:55.

37. Rübe CE, Wilfert F, Uthe D, et al. Modulation of radiation-induced tumour necrosis factor alpha (TNFalpha) expression in the lung tissue by pentoxifylline. Radiother Oncol 2002;64:177-87.

38. Jackson IL, Chen L, Batinic-Haberle I, et al. Superoxide dismutase mimetic reduces hypoxia-induced $\mathrm{O}^{*}{ }^{*}$, TGF-

Cite this article as: Ma H, Peng F, Xu Y, Bao Y, Hu X, Wang J, Fang M, Kong Y, Dong B, Chen M. Five-year survival rate analysis: the combination of fortnightly-administration of endostar and concurrent chemoradiotherapy versus concurrent chemoradiotherapy in the treatment of inoperable locally advanced non-small cell lung cancer. Ann Palliat Med 2021;10(7):7560-7570. doi: 10.21037/apm-21-1092 beta, and VEGF production by macrophages. Free Radic Res 2007;41:8-14.

39. Tanabe K, Maeshima Y, Ichinose K, et al. Endostatin peptide, an inhibitor of angiogenesis, prevents the progression of peritoneal sclerosis in a mouse experimental model. Kidney Int 2007;71:227-38.

40. Zhang K, Yang S, Zhu Y, et al. Protection against acute radiation-induced lung injury: a novel role for the antiangiogenic agent Endostar. Mol Med Rep 2012;6:309-15.

41. Guan Y, Li A, Xiao W, et al. The efficacy and safety of Endostar combined with chemoradiotherapy for patients with advanced, locally recurrent nasopharyngeal carcinoma. Oncotarget 2015;6:33926-34.

42. Ke QH, Zhou SQ, Huang M, et al. Early efficacy of Endostar combined with chemoradiotherapy for advanced cervical cancers. Asian Pac J Cancer Prev 2012;13:923-6.

43. Zheng YF, Ge W, Xu HL, et al. Endostar enhances the antitumor effects of radiation by affecting energy metabolism and alleviating the tumor microenvironment in a Lewis lung carcinoma mouse model. Oncol Lett 2015;10:3067-72. 
Supplementary

Table S1 Late-stage lung injury under different chemotherapy regimens

\begin{tabular}{|c|c|c|c|c|c|c|c|c|}
\hline Chemotherapy regimens $(n=193)$ & \multicolumn{2}{|c|}{ Grade 1} & \multicolumn{2}{|c|}{ Grade 2} & \multicolumn{2}{|c|}{ Grade 3} & \multicolumn{2}{|c|}{ Total } \\
\hline EP [67] & 15 & 7.8 & 3 & 1.6 & 1 & 0.5 & 19 & 34.7 \\
\hline $\mathrm{DP} / \mathrm{C}[60]$ & 5 & 2.6 & 3 & 1.6 & 0 & 0 & 8 & 31.1 \\
\hline $\mathrm{TP} / \mathrm{C}[48]$ & 17 & 8.8 & 5 & 2.6 & 0 & 0 & 22 & 24.9 \\
\hline AP [5] & 2 & 1.0 & 0 & 0 & 0 & 0 & 2 & 2.6 \\
\hline GP [4] & 2 & 1.0 & 1 & 0.5 & 0 & 0 & 3 & 2.1 \\
\hline Total & 45 & 23.3 & 12 & 6.2 & 1 & 0.5 & 58 & \\
\hline
\end{tabular}

EP, etoposide, cisplatin; DP/C, docetaxel, cisplatin, or carboplatin; TP/C, paclitaxel, cisplatin, or carboplatin; NP, vinorelbine, cisplatin; AP, pemetrexed, cisplatin; GP, gemcitabine, cisplatin. 